This study aimed to compare respiratory variation in transthoracic echo-derived aortic blood flow velocity DVpeak and DIVCD with DVpeak and Doppler corrected flow times obtained by TOD.

Methods A prospective and comparative study conducted in pediatric intensive care unit investigated 11 mechanically ventilated children using TTE and TOD for each patient had tachycardia, hypotension, oliguria, delayed capillary refilling or hemodynamic instability despite vasopressor drugs.

Results VE induced significant changes in TTE and TOD, the DVpeak ao in responders was higher than that in non-responders [23\% (15-32.1) vs.10\% (6-14) by TTE and, [21\% (14-29) vs.12\% (11-13) by TOD, whereas DVCID and FTc did not significantly differ between groups.

Conclusion In this study, $\Delta$ Vpeak was the most appropriate variable to predict fluid responsiveness by TTE and TOD. DIVCD and FTc are of little value in ventilated children.

\section{CONVENTIONAL $V S$. RESTRICTIVE MAINTENANCE FLUID REGIME IN CHILDREN WITH SEPTIC SHOCK AFTER INITIAL RESUSCITATION: A RANDOMIZED OPEN LABEL CONTROLLED TRIAL}

doi:10.1136/archdischild-2012-302724.0015

G Benakatti, S Singhi, J Muralidharan, A Bansal. Pediatrics, Post Graduate Institute of Medical Education and Reasearch (PGIMER), Chandigarh, India

Background Initial aggressive fluid resuscitation is of proven benefit in septic shock. Optimal post resuscitation fluid management is not known.

Aims To compare restrictive vs. conventional post-resuscitation fluid protocols in children with septic shock.

Methods We performed prospective randomized trial involving children (3 to144 months) with septic shock admitted to our PICU. After initial resuscitation, patients were randomly assigned to restrictive (A) or conventional (B) fluid protocol. The primary end point was length of PICU stay. Secondary end points included: all cause mortality, organ failure free days, ventilator-free days, measures of lung physiology and incidence of AKI. All analyses were performed on intention-to-treat basis. Intergroup differences were tested with Students' t test, Chi-square and Mann Whitney U test as appropriate and ANOVA for repeated measures. Time to event data was analyzed with Kaplan-Meier method and Mantel-Cox log rank test.

Results In 12 months period, total of 101 children were enrolled. The baseline characteristics of both groups were similar. The mean $( \pm \mathrm{SD})$ cumulative fluid balance in initial ten days was - $42.6 \pm 82.6 \mathrm{ml}$ (group A) and $339 \pm 117 \mathrm{ml}$ (group B) $(\mathrm{P}<0.001)$. As compared to Group B, group A showed significantly more PICU free days [17.2 $\pm 9 \mathrm{vs} .12 .7 \pm 9.5$ days; $p=0.015]$, lesser number of organ failures $[\mathrm{p}=0.001]$, higher proportion of patients recovering from organ failure [92.5vs.75\%; $\mathrm{p}=0.005]$, improved oxygenation index and plateau pressure $[p=0.001]$, lesser duration of ventilation $[6.3 \pm 5.8$ vs.9.9 \pm 5.2 days; $p=0.012]$, early recovery from shock [92.5 \pm 68.8 vs. $123 \pm 87$ hours; $p=0.05]$. Mortality was similar [18.5vs. $23.4 \%$; $\mathrm{p}=0.54]$.

Conclusions Restrictive fluid strategy improved lung function; shortened ventilation and ICU stay without aggravating the hemodynamic instability.

\section{DELAYED CORD CLAMPING AND NEED FOR TRANSFUSIONS}

doi:10.1136/archdischild-2012-302724.0016

H Rabe. Academic Department of Paediatrics, Brighton and Sussex Medical School, Brighton, UK
The requirement of donor blood transfusions is a common problem in the treatment of preterm infants, especially below 32 weeks' gestation. Upto $50 \%$ of these infants receive at least one transfusion during their hospital stay. Studies on enhancing placento-fetal transfusion have demonstrated a significant increase in circulating blood volume in preterm infants if the cord is either not clamped immediately after birth or is milked several times. The recent updated Cochrane review showed positive benefits of placento-fetal transfusion with regard to better adaptation (e.g. higher blood pressure) after birth as well as reduced intra-ventricular haemorrhage and necrotizing enterocolitis. Infants receiving extra placental blood at birth have a reduced need for donor blood transfusions during the first six weeks of life. The talk will focus on a structured review of the available literature. In addition other preventative measures of anaemia of prematurity such as early protein and iron supplementation will be reviewed.

\section{RED BLOOD CELL TRANSFUSIONS IN CHILDREN}

doi:10.1136/archdischild-2012-302724.0017

0 Karam. Geneva University Hospital, Geneva, Switzerland

All our cells need oxygen for their metabolism. Oxygen delivery is dependant on cardiac output, hemoglobin and arterial blood saturation. Therefore, red blood cells (RBC) transfusions could seem to be the best therapy to increase oxygen delivery in critically ill children. However, RBC transfusions are associated with an increased morbidity and mortality. This might be due to the storage lesions, which decrease the stored RBC's ability to transport oxygen in the microcirculation, and modify their immunomodulative properties. Therefore, one must carefully select the patients for whom the benefits will be greater than the risks.

For unstable critically ill children, it is usually recommended to transfuse for a $\mathrm{Hb}$ threshold of $100 \mathrm{~g} / \mathrm{l}$, after correcting the cardiac output.

For critically ill children, it is recommended to transfuse for a $\mathrm{Hb}$ threshold of $70 \mathrm{~g} / \mathrm{L}$. This threshold has also been validated for septic patients as well as surgery and cardiac surgery patients. For singleventricle physiology patients, it seems reasonable to transfuse RBC units for a threshold of $90 \mathrm{~g} / \mathrm{L}$. For neonates, a higher threshold is used ( $\mathrm{Hb} 120-140 \mathrm{~g} / \mathrm{L}$ if $\mathrm{FiO} 2>40 \%$, $\mathrm{Hb} 100 \mathrm{~g} / \mathrm{L}$ if $\mathrm{FiO} 2<40 \%, \mathrm{Hb}$ 70-80 g/L for asymptomatic infants). Lower thresholds have been proposed for chronically anemic children ( $\mathrm{Hb} 50 \mathrm{~g} / \mathrm{L}$ ).

$\mathrm{RBC}$ transfusions are a common treatment, but one must be aware of the associated risks and the appropriate transfusion indications, in order to prevent unnecessary morbidity and mortality.

\section{HEAD-TO-BODY DELIVERY BY 'TWO-STEP' APPROACH: EFFECT ON UMBILICAL ARTERY HEMATOCRIT AND PH}

doi:10.1136/archdischild-2012-302724.0018

'V Zanardo, 'A Simbi, 'P Guerrini, 'I Gattolin, ${ }^{2} \mathrm{D}$ Trevisanuto, ${ }^{3} \mathrm{~V}$ Dal Cengio, ${ }^{1} \mathrm{G}$ Straface. 'Policlinico Abano Terme, Abano Terme; 'Pediatrics; ${ }^{3}$ Padua University School of Medicine, Padua, Italy

Background The timing of umbilical cord clamping has a profound effect on the amount of blood that remains in the infant's circulation at birth. However, there is no evidence to support a relationship between cord clamping time and other active management techniques of labor.

Objective To examine the association between head-to-body delivery by 'two-step' approach, that include waiting for the next contraction to deliver the shoulders, and early cord clamping $(<1 \mathrm{~min})$ and its effect on the amount of blood that remains in the infant's circulation at birth and cord artery blood gas parameters.

Study Design Prospective observational study on 50 consecutive at term, uncomplicated vaginal deliveries with singleton cephalic 